\section{Medikamentöse Ansätze für Patienten mit Alkoholproblemen in der Praxis}

Albrecht Ulmer
Zusammenfassung: In den meisten Fällen ist die Behandlung der Alkoholabhängigkeit ambulant möglich. Häufig werden dazu Medikamente benötigt. Der ambulante Entzug ist mit täglicher Sprechstunde und Mitgabe abgezählter Medikamente wie Carbamazepin oder Gabapentin und weiterer Medikamente bereits ansatzweise standardisiert. In der weiteren Behandlung enttäuschen Anticravingmittel oft, stellen aber eine wichtige Hilfe zum Aufbau einer Behandlungsstruktur dar, fast immer mit Teilerfolgen, auf denen sich aufbauen lässt. Ein nicht sofort $100 \%$ iger Erfolg bedingt oft einen langjährigen, ringenden Prozess, für den es noch kaum wissenschaftlich erprobte medikamentöse Hilfen gibt. Aber vieles ist in spezialisierten Praxen und Ambulanzen erprobt: z.B. neue Bewertungen alter Medikamente wie Disulfiram, evtl. auch Clomethiazol unter speziellem Setting. Auch Opioidbehandlungen für Alkoholabhängige, speziell mit Dihydrocodein, haben sich vereinzelt als erfolgreich erwiesen und müssen deshalb wissenschaftlich überprüft werden. Bisher sind sie spezialisierten Praxen/Zentren vorbehalten. Unter konsequenter Nutzung und Weiterentwicklung der hier vorgestellten Möglichkeiten kann einer viel größeren Zahl von Patienten mit Alkoholproblemen erheblich besser geholfen werden.

Schlüsselwörter: Alkoholabhängigkeit - Ambulante Behandlung - Praxis - Carbamazepin - Gabapentin Clomethiazol - Acamprosat - Disulfiram - Dihydrocodein

Medically-Based Approches for the Treatment of Patients with Alcohol Problems: Growing experience shows that the treatment of alcoholism on an outpatient basis can be a supplement and even an alternative to hospitalization for most patients. However, medication will often be necessary. Most detoxification treatments should be possible on an outpatient basis. A beginning standard treatment is based on daily consultations and supervision of exact dosages of carbamazepin or gabapentin and clomethiazol. In long-term treatment anticraving substances can be an enormous help in developing a trusting relationship between the doctor and patient. Although the effect of the anticraving substances is often disappointing, partial success very often gives the patient the will to continue. If the treatment is not immediately successful, the patient is faced with a very long process

Suchttherapie 2001; 2: 183-188

(c) Georg Thieme Verlag Stuttgart · New York ISSN 1439-9903 for which no scientifically proven medications are available. Spezialiced practices and outpatient departments have developed treatments using many different medications such as antidepressants and neuroleptics. When very carefully described and applied the use of substances like disulfiram and clomethiazol have shown new possibilities. In some cases, even opioid treatments for alcoholics, especially with Dihydrocodeine, have proven successful. These are presently limited to spezialized practices and centres. The broad suitability depends on continued research and structuring. If all the new possible treatments are consistently developed and applied, we should be able to help many more patients suffering from alcohol dependencies.

Key words: Alcoholism - Outpatient - Practice - Carbamazepin - Gabapentin - Clomethiazol - Acamprosat Disulfiram - Dihydrocodeine

\section{Vorbemerkung}

Medikamente sind in der Behandlung der Alkoholabhängigkeit nie das Einzige und selten das Wichtigste. Aber darauf zu verzichten hieße, wichtige Chancen von enormer Tragweite ungenutzt zu lassen. Es geht um Millionen Betroffene und der Bedarf an Innovation als Ergänzung $\mathrm{zu}$ den bisherigen Möglichkeiten ist hoch. Mit ambulant eingesetzten Medikamenten zeichnet sich eine beträchtliche Erweiterung der bisherigen Behandlungsmöglichkeiten ab, die der gesamten Suchttherapie neue Impulse verleihen kann.

Diese Chancen zeigen sich vor allem in Praxen und Ambulanzen, die sich auf die Behandlung der Alkoholabhängigkeit spezialisiert haben. Darauf wird am Ende des Beitrags eingegangen. Noch sind es wenige „Zentren“, ist wenig Vernetzung organisiert und es gibt große Unterschiede in den einzelnen Methoden und Erfahrungen. Aber es ist bereits lohnend zusammenzufassen, was sich schon als allgemeingültig erkennen lässt.

Neu zu bestimmen gegenüber Patienten mit Alkoholproblemen ist die Rolle niedergelassener Ärzte. In den meisten Publikationen der letzten Jahre [z. B. 1-3] wird ihnen überwiegend eine zutragende Rolle zum eigentlichen therapeutischen System zuerkannt. Tatsächlich ist es aber nötig, dass sie selbst ein aktiver Teil dieses therapeutischen Systems werden. 
1. Alkoholabhängigkeit und ihre Vorstufen sind oft hervorragend ambulant behandelbar und das Gros der Hilfen spielt sich ambulant ab.

2. Medikamente spielen dabei eine sinnvolle und teilweise notwendige Rolle.

3. Auf diese Weise ist ein viel pragmatischeres Rückfallmanagement möglich.

4. Für viele Patienten ist es zunächst weniger outend, regelmäßig zum Arzt zu gehen als zu speziellen Anlaufstellen für „Süchtige“ oder „Alkoholiker“.

5. Die direkte Mitbeachtung körperlicher Zusammenhänge und beispielsweise notwendiger Arbeitsunfähigkeitsbescheinigungen im Rahmen von Gesamtkonzepten kann die Behandlung verbessern.

6. Vielfach, wahrscheinlich in der Regel, muss die Behandlung der Alkoholabhängigkeit langjährig begleitend sein, ähnlich wie bei der Opiatabhängigkeit.

7. Bei konsequenter Nutzung dieser Gesichtspunkte kann viel mehr Menschen mit Alkoholproblemen geholfen werden als bisher.

Selbstverständlich ist die Mitwirkung von niedergelassenen Ärzten Teil eines interdisziplinären Verbundes, eine Ergänzung. Das ist, als niedergelassene Ärzte begonnen haben, Opiatabhängige zu behandeln, schon einmal gründlich missverstanden worden, damals als Affront gegen die etablierte Suchttherapie und die stationäre Psychiatrie. Heute haben wir solche Missverständnisse nicht mehr nötig. Es bedarf aber aktiver Vernetzungsarbeit, damit die verschiedenen Ansätze, von der Grundlagenforschung bis zur Praxis, von der Psychologie über die Medizin bis zur Selbsthilfe, zusammenfinden und sich gegenseitig befruchten.

Der vorliegende Beitrag behandelt nur einen Ausschnitt dieser Thematik: die in der Praxis verfügbaren medikamentösen Ansätze. Vor dem Hintergrund der Erfahrungen aus eigener Praxis wird versucht, heutige Möglichkeiten im Überblick darzustellen. Zuletzt wird besonders auf eine Behandlungsmethode eingegangen, mit der sich unsere Praxis speziell auseinander gesetzt hat, die Substitution mit Opioiden.

\section{Der ambulante Entzug}

Der ambulante Entzug ist eines der wichtigsten Angebote für Alkoholabhängige. Patienten reagieren auf dieses Angebot oft mit großer Erleichterung und mit einer Öffnung, die sie sonst nicht zeigen würden.

Etwa 50\% unserer Alkoholpatienten brauchen keinen ärztlich gestützten Entzug, weil sie den Alkoholgebrauch immer wieder selbst beenden können. Einen stationären Entzug halten wir nur bei weniger als $10 \%$ für nötig, vor allem immer dann, wenn sich aus Anamnese und Untersuchung ergibt, dass es ambulant zu gefährlich wäre. In unserer Praxis bleiben also etwa $40 \%$ der Alkoholpatienten übrig, denen wir, z.T. immer wieder, Medikamente zum Alkoholentzug rezeptieren.

Dabei übernehmen wir eine hohe Verantwortung. Trotz des oft relativ leichten Verlaufes sind Alkoholentzüge komplikationsträchtig und potenziell lebensgefährlich. Deshalb sind Anamnese und Untersuchung, auch zur juristischen Absicherung, so wichtig und genauso eine klare Struktur.
Für uns heißt das:

1. geplante ambulante Entzugsbehandlungen möglichst grundsätzlich - montags zu beginnen, um mit der Phase der größten Komplikationsgefahr (Tag 2-4) nicht ins Wochenende zu geraten.

2. schriftliche Information und Einverständnis des Patienten. Dazu gehören u.a.

a) Bestätigung des Patienten, dass er alkoholabhängig ist,

b) psychosoziale Hilfen besprochen zu haben,

c) dass eine Kombination der ärztlich verordneten Medikamente mit Alkohol sowie das Auslassen von Arztterminen lebensgefährlich sein kann,

d) Hinweise auf die höhere Gefahr eines ambulanten Entzuges und

e) die Verkehrsuntauglichkeit während der Entzugsbehandlung.

3. tägliche Sprechstunde mit zumindest orientierender Untersuchung des Patienten an den Werktagen.

4. täglich abgezählte Mitgabe nur der bis zum nächsten Tag benötigten Entzugsmedikamente.

Als Basismedikamente haben sich Substanzen bewährt, die ursprünglich gegen zerebrale Anfälle entwickelt wurden. Die breiteste Erfahrung besteht mit Carbamazepin (Beschreibung ambulanter Erfahrungen schon 1976 [4]). Auf dreimal täglich verteilt, am ersten Tag 450 bis $600 \mathrm{mg}$, ab Tag zwei $900 \mathrm{mg}$, kann die Dosis ab dem fünften Tag wieder reduziert werden. Da einige Patienten unter Carbamazepin einen psychostabilisierenden und Craving-reduzierenden Effekt verspüren, halten wir die Carbamazepinbehandlung normalerweise noch wochen- bis manchmal monatelang in einer Dosierung von $2 \times 300 \mathrm{mg} / \mathrm{Tag}$ bei. Dabei richten wir uns individuell nach dem Verlauf und der von den Patienten verspürten Wirkung. Retardierte Carbamazepinpräparate gelten im Alkoholentzug als obsolet, wenn man den Patienten ganze Packungen verschreibt und ihnen selbst das richtige Dosieren anvertraut. Bei täglich abgezählter Tablettenmitgabe haben Retardpräparate unseres Erachtens eher Vorteile, was aber nicht durch Studien belegt ist. Die täglich abgezählte Mitgabe praktizieren wir, indem wir ganze Schachteln verschreiben und die Patienten auffordern, diese sofort aus der Apotheke zu uns zu bringen. In aller Regel können wir uns darauf verlassen. Wenn Zweifel daran bestehen, ist es eine Organisationsfrage mit der Apotheke.

Nebenwirkungen sind sehr selten. Wegen möglicher QT-Verlängerungen und Herzrhythmusstörungen auch auf dem Boden einer alkoholbedingten Kardiomyopathie leiten wir grundsätzlich vor Einleitung der Therapie ein EKG ab. Gelegentlich wird über Schwindel, leichtere Benommenheit und etwas Übelkeit berichtet, wobei nicht immer klar ist, inwieweit diese Symptomatik vom Entzug selbst herrührt. Selten kommen Allergien vor, sehr selten auch schwere.

Bei Carbamazepin kommen über das Cytochromsystem Interaktionen mit anderen Medikamenten vor. Bei Opiatsubstituierten beispielsweise können so verstärkte Entzugserscheinungen auftreten, deren Ausgleich selbst mit einer Anpassung der Opioiddosis schwierig sein kann. Mit Gabapentin (Neurontin $^{\circledR}$ ) steht eine Alternative mit vergleichbarer Wirkung ohne Interaktionen am Cytochromsystem zur Verfügung. Es wird in gleicher Dosis wie Carbamazepin eingesetzt. 
Benzodiazepine spielen im angloamerikanischen Raum und dort in der von großen Behandlungszentren geprägten Literatur eine große, erfolgreiche Rolle [z.B. 5]. Unsere eigenen Erfahrungen mit Benzodiazepinen zum Entzug sind gering. Suchtkranke haben in großer Zahl erhebliche Dauerprobleme mit Benzodiazepinen. Verschreibungen von verschiedenen Ärzten kommen vor und auch auf dem Schwarzmarkt sind Benzodiazepine verbreitet. Deshalb ist unser Verhältnis diesen Substanzen gegenüber bisher von einer großen Zurückhaltung geprägt.

Der Verzicht auf Benzodiazepine bedeutet, dass in der Regel noch etwas gegen die zum Delir tendierende, nervliche Überstimulation gegeben werden muss, die ja ohnehin in den ersten Tagen mit gehäuften Schlafstörungen einhergeht. Dazu eignet sich Clomethiazol (Distraneurin ${ }^{\circledR}$ ) in einer Dosis von ein bis maximal vier Kapseln für den Verlauf der Nacht, immer abgezählt mitgegeben, insgesamt hervorragend. Bei höherer Dosis und Missbrauch besteht das Risiko lebensgefährlicher Intoxikationen, vor allem bei der Kombination mit Alkohol [6]. Deshalb dürfen keinesfalls ganze Schachteln auf einmal überlassen werden.

Die Gefahr einer Clomethiazol-Suchtinduzierung beobachten wir bei dem beschriebenen, strukturierten Vorgehen, bei dem dieses Medikament nach wenigen Tagen bis spätestens zwei Wochen wieder abgesetzt ist, nicht.

Zur Milderung starker vegetativer Symptome und eines starken Entzugstremors kann Tiaprid (in den ersten Tagen in der Regel 3 x $300 \mathrm{mg}$ ) eingesetzt werden. Wir überblicken erst Erfahrungen bei etwa 15 Patienten, bei denen die Wirksamkeit unterschiedlich ausgeprägt war.

Bei Hypertonie kann gleichzeitig Clonidin eingesetzt werden, das eine ähnlich lindernde Funktion auf Entzugsbeschwerden haben kann. Die dafür erforderlichen Dosierungen erscheinen uns beim ambulanten Setting aber problematisch. Wir haben die Dosis von $3 \times 0,075 \mathrm{mg}$ vorsichtshalber bisher nicht überschritten. Eine tägliche Blutdruckmessung empfiehlt sich ohnehin.

Eine Magnesium- und Vitamin- $\mathrm{B}_{1}$-Substitution ist zusätzlich zu empfehlen, umso mehr, je mehr mit einem eher schweren Entzug zu rechnen ist.

Das A und $\mathrm{O}$ eines ambulanten Entzugs ist, dass er gut strukturiert durchgeführt wird, von der sorgfältigen Patientenauswahl über eine gute schriftliche und mündliche Information und Einverständnis des Patienten einschließlich schriftlicher Mitteilung von Notrufnummern, eine exakte Planung mit schriftlichen Plänen in der Hand von Arzt und Patient, tägliche Sprechstunde mit verlässlicher Beurteilung des Zustandes bis zu einer Planung über den Entzug hinaus.

Insgesamt stößt dieses Entzugsverfahren auf eine sehr gute Resonanz. Regelmäßige Befragungen der Patienten anhand einer sechsstufigen Zufriedenheitsskala ( 1 = sehr zufrieden $6=$ sehr unzufrieden) nach einer Woche ergeben in etwa $90 \%$ die Bewertungen eins bis drei und noch etwas bessere Bewertungen bei der Frage nach dem aktuellen Suchtdruck der letzten Tage. Eine Krankschreibung ist nicht immer nötig, aber in der Regel für ein bis besser zwei Wochen angezeigt. Unsere Abbruchquote liegt bei $6 \%$.

Die Kenntnis strukturierter, ambulanter Entzüge muss sich dringend ausbreiten. Sie wird sehr viel mehr als bislang verfügbar gebraucht.

\section{Nach dem Entzug}

Ohne weitere Therapie besteht eine hohe Rezidivquote von über $90 \%$. Da das Thema dieses Beitrags nur die medikamentöse Behandlung ist, sei hier lediglich angedeutet, dass eine weitere Auseinandersetzung mit der Alkoholsucht über Beratungsstellen und Selbsthilfegruppen zwar nicht für jeden Patienten zwingend einzufordern ist, aber von Beginn an das Regelangebot darstellt und für die Mehrheit der Patienten auch eine unverzichtbare Hilfe bedeutet. Auch für den Arzt stellt eine gute Kooperation eine unersetzliche Hilfe dar.

Zur Behandlung in der Praxis gehören, wie beim Diabetes, die Langfristigkeit und besonders die Betreuung der Patienten mit nicht völlig erfolgreichen Verläufen. 100\%ige Erfolge sind die Ausnahme. Überwiegend haben wir es mit Teilerfolgen zu tun. Es macht einen entscheidenden, innovativen Unterschied aus, mehr die Erfolge als die Misserfolge zu fokussieren und neu zu definieren. Eindeutig zeigt sich, dass „weniger trinken“ zählt und man darauf aufbauen kann. Natürlich gilt das mehr im Sinne eines Prozesses und weniger statisch, und es ist an die Bedingungen eines ehrlichen, offenen Ringens geknüpft, für das eine oft jahrelange Betreuung einen guten Boden bereiten kann.

Berücksichtigt werden muss nicht nur die Reduktion des Alkoholgenusses, sondern die gesamte körperliche und psychische Situation. Dysphorie, Initiativblockaden und alle weiteren Arten psychischer und sozialer Störungen können das Gesamtbild im Einzelfall weit mehr beeinträchtigen als der konkrete Alkoholkonsum.

Zunächst geht es aber darum, dass der Alkohol so wenig wie möglich Leben und Pläne durchkreuzt. Praktisch alle Patienten, die sich wegen Alkoholproblemen in unserer Behandlung befinden, brauchen Hilfe zum Nicht-wieder-zu-viel-Trinken. Hierfür Medikamente einzusetzen begründet sich unter anderem durch die Erkenntnis, dass die Abstinenzquoten aller ambulanten therapeutischen Bemühungen ohne medikamentöse Hilfe insgesamt enttäuschend sind. In der deutschen Acamprosat-Studie [7] lag sie nach einem Jahr bei strenger Auswertung, die jeden kleinsten Rückfall als „Nicht-Abstinenz" wertete, bei etwa $10 \%$, nach zwei Jahren sogar unter $5 \%$.

In dieser Studie konnte bei doppelblindem Design eine Verdoppelung der Abstinenzrate durch Acamprosat gezeigt werden. Diese Verdoppelung ist absolut nicht viel. Sie kann die Tatsache überwiegender Wirkungslosigkeit nicht beseitigen, und bei Naltrexon, der zweiten (noch nicht in Deutschland) zugelassenen Anticravingsubstanz [8], sind die Ergebnisse eher noch schlechter.

Trotzdem ist Acamprosat eine große Hilfe. Nach unserem Eindruck trägt es oft entscheidend zum Entstehen einer geordneten Behandlungsstruktur und einer therapeutischen 
Beziehung bei. Der Einsatz eines Medikamentes an sich bedeutet in der Praxis viel. Man geht kaum immer wieder zum Arzt, um sich nur Ratschläge zu holen. Bei etwa $20 \%$ zeigen sich beeindruckende Erfolge, wo die Einhaltung von Abstinenz in einer Weise gelingt wie vorher lange nicht. Hinzu kommen Teilerfolge, etwa, dass „Rückfälle“ viel glimpflicher verlaufen als sonst immer. Im Einzelfall kann solch konkretes „Weniger-Trinken“ von entscheidender Bedeutung sein. Acamprosat besitzt kein eigenes Abhängigkeitspotenzial und keine nennenswerten Nebenwirkungen. So ist es in der Summe der Abwägungen für uns das Medikament der ersten Wahl nach dem Entzug und wird in der Regel eingesetzt.

In Studien wurden 6- und 12-Monats-Regimes erprobt. Das ist auch die Empfehlung, mit der Acamprosat zugelassen ist. Wir haben 1998 die Verläufe von 98 eigenen Patienten ausgewertet. Nur 19 (19,4\%) von ihnen haben Acamprosat, wie empfohlen, ein Jahr lang durchgehend eingenommen. Mehrjährige Katamnesestudien liegen bisher nicht vor. Die Reichweite der Methode ist also begrenzt.

Weitere Medikamente mit wissenschaftlich erprobter Wirkung zur Reduktion des Alkoholverlangens sind für die Praxis nicht verfügbar. Patienten trotzdem weiter helfen $\mathrm{zu}$ wollen, bedeutet deshalb eine besondere Herausforderung. Es gilt, Hilfen zu finden, wo es nichts oder wenig Standardisiertes gibt. Auf diese Weise haben sich die besonderen Erfahrungen spezialisierter Praxen entwickelt.

Immer wieder gehört das geduldige, pragmatische Auffangen von Rückfällen dazu. Dann ringt man darum, wie die Rückfälle minimiert und ganz vermieden werden können. Neben der psychosozialen Arbeit und dem entscheidenden Anteil, den der Patient selbst dabei leistet, erstreckt sich die ärztliche Suche auch auf eine Art Basismedikament. Es soll bezüglich der Rückfälle eine ähnliche Funktion haben wie Basismedikamente bei der Migräne, die dazu beitragen, dass Anfälle weniger häufig und weniger schwer auftreten, im Idealfall ganz verschwinden.

Einer gehäuften Rückfälligkeit liegt immer zugrunde, dass „etwas nicht stimmt“. Häufig können Patienten das differenziert schildern und es ist sinnvoll, sie genau danach zu fragen. Neben der Bedeutung, die das für die oft zentral wichtige Patienten-Arzt-Beziehung hat, kann sich auf medikamentöser Ebene so der gezielte und differenzierte Einsatz von Antidepressiva und eventuell Neuroleptika ergeben. Auch die längere Beibehaltung von Carbamazepin oder Gabapentin im Medikamentenplan hat sich daraus ergeben. Wenn aber einfach ein starkes, „unstillbares“ Verlangen nach Alkohol besteht, das für die Patienten selbst schwer erklärbar ist, ist die Hilfe auch dieser Medikamente meistens begrenzt.

In einzelnen Praxen wird bei einer großen Zahl von Patienten mit Disulfiram gearbeitet und von hervorragenden Erfolgen berichtet (z.B. [9]). Offensichtlich ist das zumindest auch die Frage eines entsprechenden, gut eingerichteten Settings. In der Literatur wird überwiegend deutlich vor Disulfiram gewarnt, insbesondere vor in Einzelfällen bedrohlichen Nebenwirkungen. Bei uns haben Behandlungsversuche bei wenigen einzelnen Patienten keine ermutigenden Resultate ergeben. Eine Disulfiram-Therapie scheint ohne spezielles Setting und ohne eingehende Beschäftigung mit den Vor- und
Nachteilen sowie den verfügbaren Alternativen für Arzt und Patient riskant (Übersicht: [10]).

Wenn Patienten immer wieder scheitern, wenn alles schon versucht wurde und keine Aussicht darauf besteht, dem Alkoholproblem in absehbarer Zeit zu entrinnen, stellt sich schließlich die Frage einer Substitution. Sie stellt sich auch, wenn bei ähnlicher Konstellation Abstinenz zwar erreicht werden kann, aber nachweislich nicht mit befriedigendem und tragfähigem Gesamtergebnis, und wenn vor ähnlichen Hintergründen massive Folgeprobleme drohen.

\section{Substitution mit Dihydrocodein}

Unter den neuen betäubungsmittelrechtlichen Regelungen dieses Jahres taucht plötzlich Dihydrocodein für Alkoholabhängige auf. Damit wird auf einen interessanten, pharmakologisch sehr sinnvollen Ansatz eingegangen, die Verordnung von Opioiden an Alkoholabhängige. Diese Idee war in der ersten Hälfte des 20. Jahrhunderts schon verbreitet [11] und dann unter den Anti-Opiat-Einstellungen und -Gesetzen, die dieses Jahrhundert weitgehend beherrscht haben, in Vergessenheit geraten. Aus der Not der Praxis wurde sie von einigen opiatsubstituierenden Ärzten wieder entdeckt, unter anderem von dem wegen seines teils grenzüberschreitenden Pioniertums umstrittenen Gorm Grimm [12].

Seine Erfahrungen und Ergebnisse wurden von Herbert Elias, dem früheren Vorsitzenden der Deutschen Gesellschaft für Drogen- und Suchtmedizin, bestätigt [13]. Auch wir können die Erfahrungen von Grimm und Elias bestätigen: Sowohl mit Dihydrocodein als auch mit Tilidin lässt sich Alkohol teilweise hervorragend substituieren. Diese Überführung eines unkontrollierten Alkoholkonsums in einen kontrollierten Konsum milder Opioide stellt zumindest bei einzelnen Patienten eine ideale Hilfe dar, die alle anderen Hilfen übertrifft.

Weil dies noch nicht etabliert und weitgehend unbekannt ist, bei richtiger Weiterentwicklung aber große Bedeutung erlangen kann, und weil unsere Praxis darin über spezielle Erfahrung verfügt, wird an dieser Stelle besonders darauf eingegangen.

\section{Zunächst einige Beispiele:}

1. Ein 45-jähriger Patient kam 1997 nach sieben stationären und drei ambulanten Entzügen sowie zwei abgeschlossenen stationären Entwöhnungstherapien zu uns. Acamprosat, parallel zu weiterer Suchtberatung, erwies sich als wirkungslos. Weitere Rückfälle zeigten, dass wir zunächst nichts erreichten. Seit November 1997 erhält er Dihydrocodein, anfangs $140 \mathrm{mg}$, seit Jahren $320 \mathrm{mg} / \mathrm{Tag}$. Seitdem ist das Craving entscheidend reduziert und kein Alkoholrückfall mehr vorgekommen.

2. Eine 38-jährige Mutter von vier Kindern kam im Januar 1997 mit einer 18-jährigen Alkoholabhängigkeitsanamnese $\mathrm{zu}$ uns. Sie hatte 22 Entzugsbehandlungen (10 stationär, 12 ambulant) und eine stationäre Entwöhnungstherapie durchgemacht und verfügte über jahrelange Erfahrungen mit Suchtberatung und Selbsthilfegruppen. Innerhalb eines Jahres wurden vier weitere Entzugsbehandlungen notwendig. Auch bei ihr war Acamprosat wirkungslos. Seit September 1998 erhält sie Dihydrocodein (250 mg/Tag) und ist seither rückfallfrei. 
3. Ihr gleichaltriger Ehemann erhält, nach einer vergleichbaren Vorgeschichte, seit Februar 1998 Dihydrocodein und ist ebenfalls seither rückfallfrei. Die Dosis konnte inzwischen von 300 auf $250 \mathrm{mg} /$ Tag reduziert werden.

4. Ein 42-jähriger Möbelverkäufer, eigentlich wegen einer fortgeschrittenen HIV-Infektion (180 Helferzellen $/ \mathrm{mm}^{3}$, orale Haarleukoplakie, reduzierter Allgemeinzustand mit mehreren Kilogramm Gewichtsverlust) gekommen, bekannte sich gleich in der ersten Sprechstunde zu einer Alkoholabhängigkeit: Seit Jahren mehrmals täglich Schnaps und Bier. In diesem Zusammenhang erhebliche psychovegetative Beschwerden und Berentungswunsch. Die GGT war auf 168 U/l erhöht. Von Oktober 1998 bis Juni 2001 wurde er mit Dihydrocodein behandelt, maximal mit 240 mg/Tag. Seit dem Beginn dieser Behandlung ist er alkoholabstinent und fühlt sich „wie ein neuer Mensch“. Er arbeitet weiter in seinem Beruf.

5. Ein 33-jähriger Patient mit schweren sozialen Problemen durch Alkohol, einer abgeschlossenen stationären Therapie, sieben stationären sowie zwei ambulanten Entzügen drohte erneut in einer Umschulung wegen exzessiver Rückfälle zu scheitern. Nach erfolgloser Acamprosat-Behandlung mit mehreren Rückfällen behandelten wir ihn von Oktober 1999 bis Februar 2001 mit Dihydrocodein, maximal $240 \mathrm{mg} /$ Tag. Darunter kam es zu einer deutlichen Stabilisierung. Die Umschulung wurde erfolgreich abgeschlossen. Der Patient befindet sich jetzt in guter beruflicher Position und ist seit der Dihydrocodeinbehandlung alkoholabstinent.

6. Bei einem 35-jährigen Männerwohnheimbewohner leiteten wir im Juli 1998 nach sechs stationären Entzügen, zwei abgeschlossenen stationären Therapien und einer erfolglosen Acamprosat-Behandlung eine DihydrocodeinBehandlung mit $250 \mathrm{mg} /$ Tag ein. Seitdem zeigt er eine deutliche Aufwärtsentwicklung: unter anderem Arbeitsaufnahme, Staplerführerschein und Umzug in eine eigene Wohnung. Es gab einzelne Alkoholrückfälle, aber keinen sozialen Rückfall und keine neue Dauerabhängigkeit. Aktuell besteht seit einem halben Jahr durchgehende Abstinenz. Als Besonderheit plädierte er von Anfang an für Tilidin statt Dihydrocodein. Er erhält es seit zwei Jahren mit zunächst $200 \mathrm{mg}$, jetzt noch $100 \mathrm{mg} / \mathrm{Tag}$.

Eine Reihe weiterer, ähnlicher Beispiele würde hier den Rahmen sprengen. Die Beispiele belegen die prinzipielle Tauglichkeit der Methode. Angesichts der Bedeutung des Alkoholproblems sollten sie, zusammen mit den Publikationen von Grimm, Elias und den Autoren Anfang des letzten Jahrhunderts, unbedingt Anlass zu weiterer Erforschung geben.

Viele praktische Fragen sind noch ungelöst. So hatten wir beispielsweise bei einzelnen Patienten den Eindruck, zu vorsichtig dosiert zu haben. Einige Patienten haben Angst vor der Opiatabhängigkeit und bleiben deshalb mit der Dosierung unter der ärztlichen Empfehlung. Spezielle Fragen stellen sich bei Patienten mit früherer Opiatabhängigkeit. Wir haben bisher bei vorsichtiger Indikationsstellung 38 alkoholabhängigen Patienten eine Dihydrocodeinbehandlung angeboten. Für echte statistische Angaben ist es noch zu früh.
Die Dihydrocodein-Substitution hat eine spezielle Geschichte in ihrem Einsatz für Opiatabhängige [14]. Zunächst sind auch dabei etliche Patienten ähnlich „aufgeblüht“ wie die hier als Beispiel geschilderten Patienten. In unserem Erleben waren es deutlich mehr als unter den heutigen, viel starrer reglementierten, überwiegend auf Methadon basierenden Substitutionsregimen. Selbst ein relativ unkontrollierter Einsatz in breiter Praxis hat zu vielfacher Schadensminderung beigetragen (Anstieg der Drogentodeszahlen seit dem Verbot), aber er ging teilweise mit nicht vertretbaren Zuständen (Massenversorgung, „Versorgung“ durch mehrere Ärzte, fehlende Dosiskontrolle, Schwarzmarkt) einher, die ihrerseits mit Todesfällen zusammenhingen. Dies und die Opiat-Sensibilität der Überwachungsbehörden führten deshalb 1998 zu einer weitgehenden Unterbindung der Dihydrocodein-Substitution. Die Sensibilität hat noch nicht nachgelassen. Gesetzgeber und Behörden würden sofort, wie bei den Opiatsubstitutionsregeln, mit praxisbehindernden Regularien reagieren, wenn sich eine breitere, wenig kontrollierte Ausbreitung dieser Behandlungsmethode für Alkoholabhängige in der Praxis zeigen würde.

Zunächst muss auf weitere Erforschung und eine gut strukturierte Etablierung mit breitem Konsens gedrungen werden. Vor einer Erprobung in nicht spezialisierter Praxis ist derzeit dringend zu warnen.

\section{Ausblick}

Die Behandlung der Alkoholabhängigkeit erhält durch die aktive Mitarbeit von niedergelassenen Ärzten neue Impulse. Auch ohne die zuletzt vorgestellte, substituierende Behandlungsmethode können wir der chronischen Alkoholkrankheit schon viel geduldiger und effektiver als allgemein bekannt und teils auf ganz neue Weise begegnen. Uns steht bereits jetzt eine Palette von Möglichkeiten und insbesondere von Substanzen zur Verfügung. Was hier aufgezählt wurde, erhebt noch keinen Anspruch auf Vollständigkeit.

Wenn die Ärzteschaft entsprechend aus- und fortgebildet und entsprechend gut organisiert ist, kann viel mehr Patienten als heute geholfen werden, auf eine ungleich pragmatischere Weise. Wir müssen es, angesichts des eigentlich Möglichen, als Skandal ansehen, dass unsere Krankenhäuser zu großen Teilen alkoholbedingt belegt sind und die dort tätigen Ärzte in der Regel weder die medizinischen Möglichkeiten, eine Rezidivprophylaxe einzuleiten, beherrschen noch spezialisierte niedergelassene Kollegen zu nennen wissen.

Das Krankheitsbild der Alkoholabhängigkeit ist so verbreitet und so bedeutend, dass es nicht reichen wird, wenn sich ein Netz spezialisierter Praxen bildet, auch wenn das ein wichtiger Anfang ist. Die hohe Verantwortung, die Ärzte insgesamt gegenüber Patienten mit Alkoholproblemen haben, muss dazu führen, dass jeder Arzt die notwendige und heute mögliche Kompetenz erwirbt, mindestens so wie die Basiskenntnis in der Diabetesbehandlung, eher noch mehr. Spezialisierte Praxen oder Zentren können, wie Facharztpraxen, nur eine Art Rückgrat bilden.

Das Problem muss grundlegend anders angegangen werden als bei der Geschichte der ärztlichen Opiatabhängigkeitsbehandlung. Dort wurde eine systematische Förderung leben- 
diger Standardisierungsbemühungen vernachlässigt. Das hat zu einer kompensatorischen Überregulierung durch Gesetze, Richtlinien und eine Fachkunde geführt, die allesamt zwar einen Mindeststandard gewährleisten, aber das Hauptproblem der Nichtbehandlung vieler Behandlungsbedürftiger $\mathrm{zu}$ wenig beheben, teilweise sogar verschärfen. Außerdem wurden wertvolle Erfahrungen spezialisierter Ärzte per Gesetz oder Richtlinien aus der Praxis verbannt.

So darf es nicht noch einmal gehen. Ärzteschaft und Politik müssen wie Industrieunternehmen aus den Fehlern der Vergangenheit lernen und ein besseres Management an den Tag legen. Dazu gehört eine intensivere, von Forschung begleitete Standardisierungsarbeit, mit starker Förderung systematischer, auch interdisziplinärer Vernetzung, und von Anfang an eine zentrale Beachtung des Problems, dass breitflächig Millionen geholfen werden muss und kann.

Die besonderen Erfahrungen spezialisierter Praxen bedeuten einen Erkenntnis- und Ideenreichtum, der viel zu wenig genutzt wird. Das gilt besonders und seit Jahrzehnten in der Suchttherapie. Praxen niedergelassener Ärzte haftet oft ein gewisses „self-made“ an, nicht zu vergleichen beispielsweise mit der professionellen Etabliertheit klassischer Suchttherapie. Die von Drittmittelforschung geprägte Universität scheint von der Ebene dieser Ideenentwicklung in der Praxis weit abgehoben und zeigt wenig Interesse.

Der Praxis wird unentwegt und zu Recht das interdisziplinäre Arbeiten gepredigt. Aber wo bleibt der genauso wichtige Austausch zwischen Praxis und Forschung? Und der zwischen der Entdeckung in der Praxis und einer breiten, strukturierten Umsetzung? Wo begleitende Forschung, Lehre und die Entwicklung angepasster Rahmenbedingungen, beispielsweise für den notwendigen Erfahrungsaustausch und die notwendige Finanzierung, ausbleiben, geht mancher gute Ansatz für die weitere Entwicklung verloren, zumindest zunächst. Und eine bloße Ausbreitung innovativer Ideen von Praxis zu Praxis geht erfahrungsgemäß schnell mit Qualitätsproblemen bis hin zu einer entstellenden Verwässerung einher.

Es ist also sehr lohnend, die in täglicher Praxis erarbeiteten Erfahrungen mit besonderer Systematik aufzunehmen, ständiger Evaluation und einer guten breiten Nutzung zuzuleiten. Für die neuen Erfahrungen und Ideen zur Behandlung der Alkoholabhängigkeit gilt das besonders.

\section{Literatur}

${ }^{1}$ Kremer G, Wienberg G, Pörksen N. Patienten mit Alkoholproblemen beim Hausarzt. MMW 1999; 141: 133-136 (11)

2 Paul A. Hausarzt in der Schlüsselposition. Über den richtigen Umgang mit Alkoholkranken. Fortschr Med 1998; 116: (2223): 49-50

${ }^{3}$ Theiß G. Gemeinsam gegen die Alkoholerkrankung. Z Allg Med 2001; 77: 134-136

${ }^{4}$ Bjorkqvist SE, Isohanni M, Makela R, Malinen L. Ambulant treatment of alcohol withdrawal symptoms with carbamazepine: a formal multicentre double blind comparison with placebo. Acta psychiatr Scand 1976; 53: 333-342 (5)

${ }^{5}$ Myrick H, Anton RF. Clinical management of alcohol withdrawal. CNS Spectr 2000; 5: 22-32 (2)

${ }^{6}$ Deutsches Ärzteblatt. Warnende Hinweise zur Verschreibung von Clomethiazol (Distraneurin). Diskussionsbeiträge und Autorenschlusswort zum Beitrag von D. Färber und R. Tölle in 33/96. Dt Ärzteblatt 1997; 94: A237-243 (5)

${ }^{7}$ Sass H, Soyka M, Mann K, Zieglgänsberger W. Relapse prevention by acamprosate: results from a placebo-controlled study on alcohol dependence. Arch Gen Psychiatry 1996; 53: 673-680

${ }^{8}$ Volpicelli JR, Clay KL, Watson NT, O'Brien CP. Naltrexone in the Treatment of Alcoholism: Predicting Response to Naltrexone. J Clin Psychiatry 1995; 56: 39-44 (Suppl. 7)

${ }^{9}$ Meyer T. Medizinische Behandlung der Alkoholkrankheit im ambulanten Bereich. Kongressband des 5. Suchtmedizinischen Kongresses der DGDS 1996: 105-110

${ }^{10}$ Berup C, Kaiser A, Jensen E. Long-term Antabuse treatment: tolerance and reasons for withdrawal. Acta Pychiatr Scand 1992; 86: 47-49

${ }^{11}$ Kafemann. Der Arzt, die Bekämpfung des Alkoholismus und die Alkoholgefährdeten. Med Welt 1929; 8: 272-273

${ }^{12}$ Grimm G. Drogen gegen Drogen. Veris. Kiel, 1992: 32-38

${ }^{13}$ Elias H. Medizinische Behandlung der Alkoholkrankheit im ambulanten Bereich. In: Zerdick J. 5. Suchtmedizinischer Kongress der DGDS (Hrsg). Kongressband, 1996: 119-124

${ }^{14}$ Ulmer A. Die Dihydrocodein-Substitution. Darstellung der Behandlungsmethode anhand von Fragen des Bundessozialgerichtes und eines Entwurfes zur achten Betäubungsmittelrechts-Änderungsverordnung. Thieme. Stuttgart, 1997

Dr. med. Albrecht Ulmer

Schwabstraße 26

70197 Stuttgart

E-mail: Dr-A.Ulmer@gmx.de 